\title{
Insights into the metabolic characteristics of aminopropanediol analogues of SYLs as S1P1 modulators: from structure to metabolism to druggability
}

\author{
Jinping Hu, Manman Zhao, Jiaqi Mi, Baolian Wang, Li Sheng, Hui Chen, Yan Li
}

State Key Laboratory of Bioactive Substance and Function of Natural Medicines, Beijing Key Laboratory of NonClinical Drug Metabolism and PK/PD study, Beijing Key Laboratory of Active Substances Discovery and Drug Ability Evaluation, Institute of Materia Medica, Chinese Academy of Medical Sciences \& Peking Union Medical College, China

Background

SYL927 and SYL930, two aminopropanediol analogues, are novel Sphingosine-1-phosphate receptor 1 (S1P1) modulators with higher selectivity and pharmacological activity compared with FTY720. Although the immunosuppressive activity of SYLs has been well demonstrated, information regarding the metabolic fates of the two chemicals is limited except for the CYP-catalyzed hydroxylation of SYL930. In this study, it was well investigated the biotransformation schemes of the two promising chemicals and the relevant molecular mechanism.

Methods

The enzyme kinetic assay for the metabolites of SYLs was conducted in rat and human liver microsomes. CYP450 isoforms and other enzymes involved in the metabolism of SYLs were identified using liver microsomes, recombinant human CYPs and liver S9 fractions. Moreover, the relevant molecular mechanism of the biotransformation of SYLs were assessed using ligand-enzyme docking analysis (CDOCKER).

Results

The hydroxylation at alkyl chain on oxazole ring by the action of CYPs was found for both SYLs in vivo. CYP2J2 and 3A4, together with CYP1A1 and CYP4F2 were observed to involved in the hydroxylation at the ethyl group of SYL927. But the SULT-catalyzed sulfonation of the hydroxide was only observed for SYL927 mediated by SULT1A1, 1A2 and 1E1, while the ADH/ALDH-catalyzed oxidation was just discovered for SYL930. The docking analysis suggested that specific noncovalent forces and/or bonding conformations of the hydroxides with biomacromolecules might be involved in the disparate metabolism of SYLs.

Conclusions

Similar hydroxylation reaction at alkyl group of the oxazole ring and different sulfonation or secondary oxidation were found for two aminopropanediol analogues of SYL927 and SYL930 as S1P1 modulators. CYPs involved in the primary hydroxylation and ADH/ALDH might mediate the carboxylation oxidation of SYL930. Due to the different biotransformation patterns, probable drug combination scheme and potential risks of drug-drug interactions in humans will be differentiated for the two chemicals. Exploring the underlying metabolic characteristics of two drugs will help clarify the substance base for efficacy and safety, and also predict the druggability based on metabolism. The uncovered structure-metabolism relationship in this study may provide an implication for the design and optimization for other S1P modulators. 